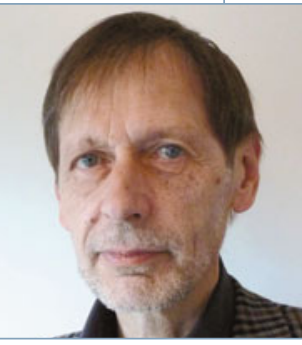

Wolfgang Nellen

\title{
Die Puddingprobe des Koalitionsvertrags für die deutsche Wissenschaft
}

DOI: $10.1007 / \mathrm{s} 12268-014-0396-3$

(C) Springer-Verlag 2014

Was früher die „Nagelprobe“ war, ist heute die „Puddingprobe“ - denn: „The proof of the pudding is the eating“, so verkündete es Bundeskanzlerin Angela Merkel nach Vorlage des Koalitionsvertrags in Anlehnung an ein altes englisches Sprichwort. Und weiter: „Zum Schluss werden uns die Menschen fragen: Geht es Deutschland in einigen Jahren besser als heute?". Auch die Akteure aus Bildung und Wissenschaft und mit ihnen die Biowissenschaftler werden sich das fragen. Und daher hat der VBIO den Koalitionsvertrag kritisch geprüft. Um das Ergebnis vorwegzunehmen - der Vertrag enthält Licht und Schatten: richtige Erkenntnisse neben ungenutzten Chancen und interessante Effekte, die den Koalitionären möglicherweise nicht voll bewusst waren. Da wäre z. B. das Kapitel "Gute Arbeit“, in dem unter anderem der gesetzliche Mindestlohn von 8,50 Euro festgelegt wird. Dieser gilt dann auch im Arbeitsmarkt „Wissenschaft“, wo es immer noch Hochschulen geben soll, die ihren studentischen Hilfskräften weniger zahlen. Konsistent wäre es dann auch, die festgeschriebene Tariftreue im Vergaberecht sinngemäß auch für öffentlich finanzierte Forschungsprojekte durchzusetzen.

Immerhin thematisiert der Koalitionsvertrag das Problem der Karrierewege in der Wissenschaft, die zukünftig planbar und verlässlich sein sollen. Sicher, die Politik ist hier nur bedingt zuständig; ein bloßer Appell an Hochschulen und Forschungseinrichtungen, in ihrer Rolle als Arbeitgeber „gegenzusteuern“, ist dann aber doch zu wenig. Wenn sich die Koalitionäre schon mangels Zuständigkeit auf die appellative Ebene begeben, so wäre ein Bekenntnis zu einem Wissenschaftstarifvertrag nebst freundlicher Aufforderung an die Tarifpartner schon eher eine Sondermeldung wert gewesen. Originäre Aufgabe der Politik ist es allerdings, Rahmenbedingungen $\mathrm{zu}$ schaffen, die den Arbeitsmarkt Wissenschaft insbesondere für Nachwuchswissenschaftler attraktiv machen. Die (nicht nur vom VBIO) mehrfach angemahnte und nun in Aussicht gestellte Novellierung des Wissenschaftszeitvertragsgesetzes ist daher notwendig, aber unter Fachpolitikern aller Parteien offenbar ohnehin Konsens. Ob sich die Koalitionäre aber bei der konkreten inhaltlichen Ausgestaltung einig sind, muss sich erst noch erweisen.

Dass der Koalitionsvertrag dazu keine konkrete Antwort gibt, ist nachvollziehbar. Es geht schließlich nicht um Details, sondern um die großen Linien, und ein Koalitionsvertrag ist auch kaum der angemessene Ort, inhaltliche Differenzen darzulegen. Dies sollte man auch im Hinterkopf behalten, wenn es um weitere Bekenntnisse der Koalitionäre zur überragenden Bedeutung von Bildung und Wissenschaft für die Zukunft Deutschlands geht. Bei näherem Betrachten drängt sich nämlich der Eindruck auf, dass im Wesentlichen die ohnehin bestehende Beschlusslage bekräftigt wird.

Egal ob Exzellenzinitiative, Hochschulpakt oder Drei-Prozent-Ziel für Forschung und Entwicklung, die Chance, bestehende Förderprogramme auszubauen und neue innovative Projekte zu initiieren, wird nicht genutzt. Schade! Trotz anderslautender Auskünfte der Parteien vor der Wahl (siehe Wahlprüfsteine unter www.vbio.de) haben die Koalitionspartner offensichtlich auch nicht den Mut gehabt, das Kooperationsverbot zwischen Bund und Ländern im Bereich Bildung und Ausbildung durch eine Grundgesetzänderung abzuschaffen. Entsprechende Überlegungen der zuständigen Arbeitsgruppe fehlen in der Endfassung des Koalitionsvertrags. Die Stichworte „Kooperationsverbot“ und „Grundgesetzänderung“ sucht man im Koalitionsvertrag vergeblich.
Konsequent gestrichen wurden in der endgültigen Version des Koalitionsvertrags auch Aussagen zu Finanzbedarf und Finanzierungsinstrumenten - obwohl es da in der Arbeitsphase durchaus die eine oder andere Idee gegeben hatte. Dass sich die Koalition in Schweigen hüllt, mag in Zeiten knapper Kassen verständlich sein, weckt aber Zweifel an der Ernsthaftigkeit mancher Aussage.

Sicher, es hätte aus Sicht der Biowissenschaften auch schlimmer kommen können; Stichworte sind hier das tierschutzrechtliche Verbandsklagerecht oder das opt-out Deutschlands bei EU-Regelungen zur Grünen Gentechnik. Aber die Merkel'sche „Puddingprobe" im Bereich Wissenschaften steht eben auch noch aus. Als VBIO werden wir darum das wissenschaftspolitische Wirken der Koalitionäre in den nächsten Jahren kritisch begleiten. Das dürfte mitunter sehr mühsam werden - aber nicht unmöglich, wie der ebenfalls sprichwörtliche Versuch, „einen Pudding an die Wand zu nageln“.

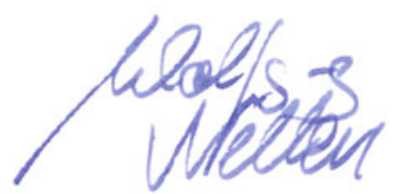

Wolfgang Nellen,

Präsident Verband Biologie,

Biowissenschaften und Biomedizin e. V. VBIO

Korrespondenzadresse: Prof. Dr. Wolfgang Nellen Abteilung Genetik Universität Kassel Heinrich-Plett-Straße 40 D-34132 Kassel Tel.: 0561-8044805 Fax: 0561-804-4800 nellen@uni-kassel.de 